\title{
Jackknife empirical likelihood inference for the mean
}

\section{absolute deviation}

\author{
Yichuan Zhao ${ }^{1}$, Xueping Meng ${ }^{2}$ and Hanfang Yang* \\ 1,2 Department of Mathematics and Statistics, Georgia State University, Atlanta, GA 30303 \\ * School of Statistics, Renmin University of China, Beijing, China \\ email: hyang@ruc.edu.cn
}

May 17, 2015

\begin{abstract}
In statistics a mean absolute deviation plays an important role in measuring spread of a data. In this paper, we focus on using the jackknife, the adjusted and the extended jackknife empirical likelihood methods to construct confidence intervals for the mean absolute deviation of a random variable. The empirical log-likelihood ratio statistic is derived whose asymptotic distribution is a standard chi-square distribution. The results of simulation study show the comparison of the average length and coverage probability by using jackknife empirical likelihood methods and normal approximation method. The proposed adjusted and extended jackknife empirical likelihood methods perform better than other methods, in particular for skewed distributions. We use real data sets to illustrate the proposed jackknife empirical likelihood methods.
\end{abstract}

KEY WORDS: Confidence interval, Jackknife empirical likelihood, Mean absolute deviation. 


\section{Introduction}

A mean absolute deviation is one of the important measures of statistical dispersion. It is a mean of the absolute deviations of a set of data about the data's mean, which is the average distance of the data set from its mean. It is closely related to the mean squared error (MSE) method which is just the average squared error. Because it is a simpler measure of variability than the standard deviation, it is an alternative tool to the standard deviation, and has some advantage such as robustness compared to the standard deviation. Some other useful measures are average absolute deviation about median, median absolute deviation (MAD) and the maximum absolute deviation about a point, etc.

If $F(x)$ is a cumulative distribution function, and $X_{1}, X_{2} \ldots X_{n}$ form i.i.d. random variables with the mean $\mu$ and variance $\sigma^{2}$. We study a mean absolute deviation as a good measure of variablility:

$$
\theta=\int_{-\infty}^{\infty}|x-\mu| \mathrm{d} F(x)=E|X-E(X)|
$$

and define $\hat{\theta}$ as an empirical estimator of $\theta$.

$$
\hat{\theta}=n^{-1} \sum_{i=1}^{n}\left|X_{i}-\bar{X}\right|,
$$

where $\bar{X}=n^{-1} \sum_{i=1}^{n} X_{i}$. For the parametric normal distribution, Herrey (1965) investigated the performance of the empirical estimator using extensive simulation studies.

The asymptotic normality of $\hat{\theta}$ is given by Gastwirth (1974).

$$
n^{\frac{1}{2}}(\hat{\theta}-\theta) \rightarrow N\left(0, v^{2}\right)
$$

where

$$
v^{2}=4 *\left\{p^{2} \int_{\mu}^{\infty}(x-\mu)^{2} \mathrm{~d} F(x)+(1-p)^{2} \int_{-\infty}^{\mu}(x-\mu)^{2} \mathrm{~d} F(x)-\frac{\theta^{2}}{4}\right\}
$$


and

$$
p=F(\mu)
$$

Let $\hat{v}$ be an empirical estimator of $v$. Then we construct a $100(1-\alpha) \%$ normal approximation (NA) based confidence interval for $\theta$ :

$$
R=\left\{\theta: \hat{\theta} \pm Z_{\alpha / 2} * \hat{v} / \sqrt{n}\right\}
$$

where $Z_{\alpha / 2}$ is the upper $\alpha / 2$ quantile of $N(0,1)$.

The NA based confidence interval for the mean absolute deviation has worse small sample performance when the data are skewed from the simulation study (see Table 2). In the paper, we develop new empirical likelihood methods for the mean absolute deviation to achieve better small sample performance. Since Owen (1988) derived the asymptotic $\chi^{2}$ distribution of empirical likelihood (EL) ratio statistic for the mean $\mu$, there have been many important contributions to EL methods in mainstream statistics. Qin and Lawless (1994) proposed an EL for a parameter solved by general estimating equations, which established the Wilks theorem. Ren (2008) worked on the two-sample problem using EL. Recently censored linear regression models have been extensively discussed by Zhao and Huang (2007), Zhao (2011) and Zhou and Li (2008), etc. More recently, Tsao (2013) proposed the extended EL procedure for general estimating equations.

Empirical likelihood has been widely utilized in many settings. However, there exist a lot of computational difficulties when applied to complicated nonlinear functional. To overcome the computational difficulties, a modified EL method was proposed by Jing et al. (2009), which was called jackknife empirical likelihood (JEL). The main idea of the JEL is to "turn the statistic of interest into a sample mean based on jackknife pseudovalues" [see Quenouille (1956)]. The jackknife empirical likelihood method is a simple application of EL which simplifies the computation for the complicated statistics, such as ROC curve, partial area under the ROC curve among others [see Gong et al. (2010), Adimari and Chiogna (2012), Yang and Zhao (2013) and Zhong and Chen (2014)]. 
The rest of the paper is organized as follows. In Section 2, we propose a jackknife empirical likelihood, an adjusted jackknife empirical likelihood and an extension of the jackknife empirical likelihood by expanding its domain for the mean absolute deviation, finding better interval estimators of the mean absolute deviation. In Section 3, we carry out the simulation study. The coverage probability and average length for all confidence intervals are calculated using all three different types of JEL and compare them to the standard normal approximation method. In Section 4, we illustrate the proposed JEL methods by real data sets. Finally, we make a conclusion and discuss possible future work.

\section{Inference Procedure}

In this section we develop new interval estimators using jackknife EL methods.

\subsection{Jackknife empirical likelihood}

We plug in the estimator (1.2) using JEL method:

$$
\hat{\theta}_{n-1}^{(-i)}=\frac{1}{(n-1)} \sum_{j \neq i}^{n}\left|X_{j}-\bar{X}_{(-i)}\right|,
$$

where $\bar{X}_{(-i)}=\frac{1}{n-1} \sum_{j \neq i}^{n} X_{j}$. The equation (2.1) indicates $\hat{\theta}_{n-1}^{(-i)}=\hat{\theta}\left(X_{1}, X_{2}, \ldots X_{i-1}, X_{i+1}, \ldots X_{n}\right)$ by removing the $i$-th item. We define our jackknife pseudo-values by:

$$
\widehat{V}_{i}=n \hat{\theta}-(n-1) \hat{\theta}_{n-1}^{(-i)}
$$

The jackknife estimator of $\hat{\theta}$ is the average of pseudo-values defined as follows:

$$
\widehat{\hat{\theta}_{n, j a c k}}=\frac{1}{n} \sum_{i=1}^{n} \widehat{V}_{i} .
$$

We display the regularity conditions given by Gastwirth (1974). 
A.1: The density function $f(t)$ is continuous in the neighborhood of $\mu$;

A.2: Mean $\mu$ and variance $\sigma^{2}$ of $X_{i}$ exist.

Lemma 2.1. Under the above regularity conditions, we have

$$
n^{\frac{1}{2}}\left(\widehat{\hat{\theta}_{n, j a c k}}-\theta\right) \rightarrow N\left(0, v^{2}\right)
$$

Then, we define the jackknife variance estimate $\sigma_{n, j a c k}^{2}=\frac{1}{n} \sum_{i=1}^{n}\left(\hat{V}_{i}-\theta\right)^{2}$.

Lemma 2.2. Under the regularity conditions, one has

$$
\sigma_{n, j a c k}^{2} \stackrel{\mathcal{P}}{\longrightarrow} v^{2}
$$

Let $P=\left(P_{1}, P_{2}, \ldots P_{n}\right)$ be the probability vector, we have that: $\quad \sum_{i=1}^{n} P_{i}=1$ and $P_{i}>0$ for $1 \leq i \leq n$. Then following Owen (1988, 1990, 2001) and Qin and Lawless (1994), one can define the jackknife empirical likelihood ratio at $\theta$ by:

$$
R(\theta)=\max \left\{\prod_{i=1}^{n}\left(n P_{i}\right): \sum_{i=1}^{n} P_{i}=1, \sum_{i=1}^{n} P_{i}\left(\hat{V}_{i}-\theta\right)=0, \quad P_{i}>0\right\}
$$

By using the Lagrange multipliers method, we have

$$
\log R(\theta)=-\sum_{i=1}^{n} \log \left\{1+\lambda\left(\widehat{V}_{i}-\theta\right)\right\}
$$

where $\lambda$ satisfies

$$
f(\lambda)=\frac{1}{n} \sum_{i=1}^{n} \frac{\widehat{V}_{i}-\theta}{1+\lambda\left(\widehat{V}_{i}-\theta\right)}=0
$$

Denote $l(\theta)=-2 \log R(\theta)$. We establish the following Wilk's theorem and show how to construct confidence interval for $\theta$. Let $\theta_{0}$ be the true value of $\theta$.

Theorem 1: Assume the above regularity conditions hold. $l\left(\theta_{0}\right)$ converges in distribu- 
tion to $\chi^{2}$, where $\chi^{2}$ is a chi-square random variable with 1 degree of freedom.

An asymptotic 100(1- $\alpha) \%$ JEL confidence interval can be constructed with the above theorem:

$$
R^{J}=\{\theta:-2 \log R(\theta) \leq C\},
$$

where $\mathrm{C}$ is chosen to satisfy $P\left(\chi^{2} \leq C\right)=1-\alpha$.

\subsection{Adjusted jackknife empirical likelihood}

Chen et al. (2008) developed an adjusted empirical likelihood method, which significantly improves the performance of the empirical likelihood method in terms of coverage probability when the sample size is not large. We adapt their approach to the JEL for $\theta$. The adjusted AJEL function for fixed $\theta$ is defined to be as Chen et al. (2008) did:

$$
L(\theta)=\max \left\{\prod_{i=1}^{n+1} p_{i}, \text { subject to } \sum_{i=1}^{n+1} p_{i} g_{i}^{a d}(\theta)=0, \quad \sum_{i=1}^{n+1} p_{i}=1, \quad p_{i}>0\right\}
$$

where $i=1,2, \ldots, n, g_{i}^{a d}(\theta)=\left(\widehat{V}_{i}-\theta\right)$, and $g_{n+1}^{a d}(\theta)=-a_{n} \bar{g}_{n}(\theta)$. Here $a_{n}=\max (1, \log (n) / 2)$, which is recommended by Chen et al. (2008),

$$
\bar{g}_{n}(\theta)=\frac{1}{n} \sum_{i=1}^{n} g_{i}(\theta)
$$

The proposed adjusted jackknife empirical log-likelihood ratio is:

$$
\log R^{a d}(\theta)=-\sum_{i=1}^{n+1} \log \left(1+\lambda g_{i}^{a d}(\theta)\right)
$$

where $\lambda$ satisfies

$$
f(\lambda)=\sum_{i=1}^{n+1} \frac{g_{i}^{a d}(\theta)}{1+\lambda g_{i}^{a d}(\theta)}=0
$$


For the adjusted jackknife empirical likelihood method, we can combine Chen et al. (2008) and the Jing et al. (2009) to get the following Wilk's theorem.

Theorem 2: Under the regularity conditions, $l^{a d}\left(\theta_{0}\right)=-2 \log R^{a d}\left(\theta_{0}\right)$ converges in distribution to $\chi^{2}$.

For the adjusted jackknife empirical likelihood (AJEL) method, an asymptotic 100(1a) $\%$ confidence interval for $\theta$ can be constructed with the above theorem:

$$
R^{a d}=\left\{\theta: l^{a d}(\theta) \leq C\right\}
$$

where $\mathrm{C}$ is chosen by $P\left(\chi^{2} \leq C\right)=1-\alpha$.

\subsection{Extended jackknife empirical likelihood}

Tsao (2013) proposed a new EL method, which extended the empirical likelihood beyond its domain $\Theta_{n}$ by expanding its contours nested inside the domain with a similarity transformation, and enables us to escape the "convex hull constraint" on the EL in order to improve the coverage accuracy [see Tsao (2013) and Tsao and Wu (2013)]. Following Tsao and $\mathrm{Wu}(2013)$, we define $h_{n}^{c}$ by using JEL $l(\theta)$ at $\theta$ :

$$
h_{n}^{c}(\theta)=\hat{\theta}+\gamma(n, l(\theta))(\theta-\hat{\theta})
$$

where $\gamma(n, l(\theta))$ is the expansion factor

$$
\gamma(n, l(\theta))=1+\frac{l(\theta)}{2 n} .
$$

We define $s(\theta)=\left\{\theta^{\prime}: h_{n}^{c}\left(\theta^{\prime}\right)=\theta\right\}$. Thus one has

$$
h_{n}^{-c}(\theta)=\operatorname{argmin}_{\theta^{\prime} \in s(\theta)}\left\{\left|\theta^{\prime}-\theta\right|\right\} .
$$

one defines the extended jackknife EL ratio for $\theta$ as 


$$
l^{e d}(\theta)=l\left(h_{n}^{-c}(\theta)\right)
$$

Theorem 3: Suppose the regularity conditions hold. $l^{e d}\left(\theta_{0}\right)$ converges in distribution to $\chi^{2}$.

Thus, the $100(1-\alpha) \%$ EJEL confidence interval for $\theta$ is

$$
R^{e d}=\left\{\theta: l^{e d}(\theta) \leq C\right\},
$$

where $C$ is defined as before.

\section{$3 \quad$ Numerical Studies}

Based on the proposed inference procedures, two groups of simulation studies are conducted to investigate the performance of normal approximation method, JEL, adjusted JEL and extended JEL methods for the mean absolute deviation. We generate the data from normal distribution and exponential distribution, then compare the performance of 4 different methods. Here we choose nominal levels 90\%, 95\% and 99\%. The sample size is from smaller to larger: 30, 50, 100, 200 and 300. For each different sample size, the repetition is 5000 times.

We simulate data set using the standard normal distribution. In Table 1, one denotes the following notations: Normal approximation method (NA), Jackknife empirical likelihood (JEL), Adjusted Jackknife empirical likelihood (AJEL), and Extended Jackknife empirical likelihood (EJEL).

[Table 1 about here.]

From Table 1, the coverage probability of the NA confidence intervals is not satisfied when the sample size is small and moderate. However, JEL, AJEL and EJEL have much better coverage probability for the same sample size. For example, when the sample size 
$n=30$, and nominal level $=95 \%$, the coverage probability of NA confidence intervals is $81.64 \%$, coverage probability of JEL confidence intervals is $93.02 \%$, coverage probability of AJEL confidence intervals is $94.62 \%$ and coverage probability of EJEL confidence intervals is $95.12 \%$, respectively. We can see coverage probability for AJEL and EJEL are very close to nominal level 95\%, and AJEL and EJEL have better performance than JEL for the small sample size.

When the sample size is large, NA, JEL, AJEL and EJEL methods have similar performance in terms of coverage probability. For example when the sample size $n=300$, and nominal level $=95 \%$, coverage probability of NA confidence intervals is $93.82 \%$, coverage probability of JEL confidence interval is $95.12 \%$, coverage probability of AJEL confidence interval is $95.40 \%$ and coverage probability of EJEL confidence interval is $95.20 \%$.

For all the methods, the length of confidence interval becomes shorter when the sample size becomes larger. When the sample size is from moderate to large, the length of confidence interval for all the methods is very close. When the sample size is smaller, the length of the NA confidence intervals is slight shorter than other three methods due to serious under coverage problem for the NA method.

[Table 2 about here.]

We generate a data set using exponential distribution, with $\lambda=1$. From Table 2, we have similar conclusions as ones from Table 1. AJEL and EJEL methods are slightly better than JEL method when we compare these three methods for the small sample size. All the jackknife methods have better coverage probability than the normal approximation method. The average length of NA, JEL, AJEL and EJEL confidence intervals for exponential distribution follows the similar pattern as the one for normal distribution. 


\section{Real Data Analysis}

In this section, we studied two real data sets to illustrate the proposed methods in Section 3. The first data set "hotdogs" has 54 observations, which was obtained from the Data and Story Library (DASL) at Carnegie Mellon University. The second data set "discoveries" has 114 observations, which was obtained from $\mathrm{R}$ dataset package in $\mathrm{R}$ program (see R Core Team (2013)).

The data set "hot dogs" is about the results of a laboratory analysis of calories and sodium content of major hot dog brands. Researchers for Consumer Reports analyzed three types of hot dog: beef, poultry, and meat (mostly pork and beef, but up to $15 \%$ poultry meat). We choose variable sodium in sample to analysis it.

We denote Upper bound (UB) and lower bound (LB). The lower bound, upper bound and length by using the NA, JEL, AJEL and EJEL methods are obtained. From Table 3 , we can see the lengths of confidence intervals with all the methods are almost the same.

[Table 3 about here.]

The data set "discoveries" is a group of time series data. The number is the "great" inventions and scientific discoveries in each year from 1860 to 1959. In this data set, only one variable is the number of discoveries were found in each year. We can see the lengths of confidence intervals for JEL, AJEL and EJEL are longer than one for the NA method from Table 4. Thus there is no big difference among the lengthes for the JEL, AJEL and EJEL methods.

[Table 4 about here.]

\section{Discussion}

In this paper, we used three types of JEL methods to construct confidence interval for the mean absolute deviation. Lengths of confidence intervals for all the JEL, AJEL 
and EJEL methods are very close. From the computational issue, we find the AJEL method is easy and shares the very good small sample performance. Thus the AJEL may be computationally easier than the EJEL, but it can give trivial $100 \%$ confidence intervals that coincide with the parameter space sometimes in the simulation study. Under exponential distribution, the coverage probability for the NA method is far away from our expectation when the sample size is small. However, the coverage probability of confidence intervals for JEL, AJEL and EJEL methods is very close to nominal level. Bonett and Seier (2003) studied all kinds of confidence intervals for median absolute deviation based on the plug-in estimator. Our proposed JEL methods can be applied to other statistical dispersion measures such as median absolute deviation as well, after combining kernel smoothing techniques. We will investigate this challenging issue in the future.

\section{Acknowledgments}

The authors are very grateful to the three referees, Associate Editor, and Co-Editor for their useful and insightful comments which help to greatly improve the paper. Yichuan Zhao acknowledges the support from the NSF Grant, NSA Grant and RIG Grant, Georgia State University. Hanfang Yang's research is partially supported by the Fundamental Research Funds for the Central Universities, and the Research Funds of Renmin University of China (13XNF059).

\section{REFERENCES}

Adimari, G. and Chiogna, M., Jackknife empirical likelihood based confidence intervals for partial areas under ROC curves, Statistica Sinica, Vol. 22, 1457 - 1477, 2012.

Bonett, D. G. and Seier, E., Confidence Intervals for Mean Absolute Deviations, The American Statistician, Vol. 57, 233-236, 2003. 
Chen, J., Variyath, A.M. and B. Abraham, Adjusted Empirical Likelihood and Its Properties, J. Comput Graph Stat., Vol. 17, 426-443, 2008.

Gastwirth, J. L., Large Sample Theory of Some Measures of Income Inequality, Econometrica, Vol. 42, 191-196, 1974.

Gong, Yun, Peng, Liang and Qi, Yongcheng, Smoothed jackknife empirical likelihood method for ROC curve, Journal of Multivariate Analysis, Vol. 101, 1520-1531, 2010.

Herrey, E., Confidence Intervals Based on the Mean Absolute Deviation of a Normal Sample, Journal of the American Statistical Association, Vol. 60, 257-269, 1965.

Jing, B., Yuan, Q. and Zhou, W., Jackknife Empirical Likelihood, Journal of the American Statistical Association, Vol. 104, 1224-1232, 2009.

Owen, Art B., Empirical Likelihood Ratio Confidence Regions, The Annals of Statistics, Vol. 18, 90-120, 1990.

Owen, Art B., Empirical Likelihood Ratio Confidence Intervals for a Single Functional, The Annals of Statistics, Vol. 75, 237-249, 1988.

Owen, Art B., Empirical Likelihood, Chapman \& Hall/CRC, 2001.

Qin, J. and Lawless, J., Empirical Likelihood and General Estimating Equations, The Annals of Statistis, Vol. 22, 300-325, 1994.

Quenouille, M., Notes on Bias in Estimation, Biometrika, Vol. 10, 353-360, 1956.

R Core Team. R: A language and environment for statistical computing. R Foundation for Statistical Computing, Vienna, Austria. URL http://www.R-project.org/. 2013.

Ren, J., Weighted Empirical Likelihood in Some Two-sample Semi-parametric Models with Various Types of Censored Data, Annals of Statistics, Vol. 36, 147-166, 2008.

Tsao, M., Extending the Empirical Likelihood by Domain Expansion, The Canadian Journal of Statistics, Vol. 40, 1-18, 2013. 
Tsao, M. and Wu, F., Empirical Likelihood on the Full Parameter Space, Annals of Statistics, Vol. 41, 2176-2196, 2013.

Wang, X., Empirical Likelihoood with Applications, Ph.D Thesis in National University of Singapore, 2010.

Yang, H. and Zhao, Y., Smoothed jackknife empirical likelihood inference for the difference of roc curves, Journal of Multivariate Analysis, Vol. 115, 270-284, 2013.

Zhao, Y. and Huang Y., Test-based Interval Estimation Under the Accelerated Failure Time Model, Communications in Statistics-Simulation and Computation, Vol. 36, 593-605, 2007.

Zhao, Y., Empirical Likelihood Inference for the Accelerated Failure Time Model, Statistics and Probability Letters, Vol. 81, 603-610, 2011.

Zheng, M. and Yu, W., Empirical Likelihood Method for Multivariate Cox Regression, Comput Stat, Vol. 138, 915-928, 2012.

Zhong, Ping-Shou and Chen, Sixia, Jackknife empirical likelihood inference with regression imputation and survey data, Journal of Multivariate Analysis, Vol. 129, 193-205, 2014.

Zhou, M. and Li, G., Empirical Likelihood Analysis of the Buckley-James Estimator, Journal of Multivariate Analysis, Vol. 99, 1069-1112, 2008.

\section{A Appendix: Proofs of Theorems}

Proof of Lemma 2.1: From the delete-one sample $\mathbf{X}_{i}=\left\{X_{1}, X_{2}, \ldots X_{i-1}, X_{i+1}, \ldots X_{n}\right\}$, we have $\bar{X}_{(-i)}=\frac{1}{n-1} \sum_{j \neq i}^{n} X_{j}, \bar{X}-\bar{X}_{(-i)}=\left(X_{i}-\bar{X}\right) /(n-1)$ and $\sum_{i=1}^{n}\left\{\bar{X}-\bar{X}_{(-i)}\right\}=0$, where

$$
\bar{X}-\bar{X}_{(-i)}=O_{p}\left(n^{-1}\right) .
$$


Using the same methods as those in Gastwirth (1974), denote $N(\mathbf{X})$ as the number of elements in the set $\left\{X_{j}<\bar{X}, j=1, \ldots, n\right\}$ and $N\left(\mathbf{X}_{i}\right)$ as the number of elements in the set $\left\{X_{j}<\bar{X}_{(-i)}, j=1, \ldots, n, i \neq j\right\}$. Further, we define $N\left(\triangle \mathbf{X}_{i}\right)$ as the number of elements between $\bar{X}$ and $\bar{X}_{(-i)}$, i.e., the set $\left\{X_{j} \in\left(\bar{X}, \bar{X}_{(-i)}\right) \cup\left(\bar{X}_{(-i)}, \bar{X}\right), j=1, \ldots, n\right\}$. Hence, we can obtain that

$$
\triangle N\left(\mathbf{X}, \mathbf{X}_{i}\right)=N(\mathbf{X})-N\left(\mathbf{X}_{i}\right)= \begin{cases}1-N\left(\triangle \mathbf{X}_{i}\right), & X_{i} \leq \bar{X} \\ N\left(\triangle \mathbf{X}_{i}\right), & X_{i}>\bar{X}\end{cases}
$$

From $\bar{X}-\bar{X}_{(-i)}=O_{p}\left(n^{-1}\right)$ and arguments in Gastwirth (1974), $N\left(\triangle \mathbf{X}_{i}\right)$ is approximately $n f(\mu)\left|\bar{X}-\bar{X}_{(-i)}\right|$, i.e.,

$$
\begin{aligned}
& N\left(\triangle \mathbf{X}_{i}\right) \\
= & n f(u)\left|\bar{X}-\bar{X}_{(-i)}\right|+o_{p}\left(\left|\bar{X}-\bar{X}_{(-i)}\right| n\right) \\
= & O_{p}(f(\mu)) .
\end{aligned}
$$

Based on the above equations, $N\left(\triangle \mathbf{X}_{i}\right)\left|\bar{X}-\bar{X}_{(-i)}\right|=O_{p}\left(f(\mu) n^{-1}\right)=O_{p}\left(n^{-1}\right)$. Consider that

$$
\sum_{X_{j}<\bar{X}} X_{j}-\sum_{X_{j}<\bar{X}_{(-i)}} X_{j}= \begin{cases}X_{i}-\sum_{\bar{X}<X_{j}<\bar{X}_{(-i)}} X_{j}, & X_{i} \leq \bar{X} \\ \sum_{\bar{X}_{(-i)}<X_{j}<\bar{X}} X_{j}, & X_{i}>\bar{X}\end{cases}
$$

If $X_{i} \leq \bar{X}$, we have $N\left(\triangle \mathbf{X}_{i}\right) \bar{X}<\sum_{\bar{X}<X_{j}<\bar{X}_{(-i)}} X_{j}<N\left(\triangle \mathbf{X}_{i}\right) \bar{X}_{(-i)}$, and

$$
\left|N\left(\triangle \mathbf{X}_{i}\right) \bar{X}-\sum_{\bar{X}<X_{j}<\bar{X}_{(-i)}} X_{j}\right| \leq N\left(\triangle \mathbf{X}_{i}\right)\left|\bar{X}-\bar{X}_{(-i)}\right|=O_{p}\left(n^{-1}\right) .
$$

Hence, with similar arguments in the case $X_{i}>\bar{X}$, we know that

$$
\begin{cases}\sum_{\bar{X}<X_{j}<\bar{X}_{(-i)}} X_{j}=N\left(\triangle \mathbf{X}_{i}\right) \bar{X}+O_{p}\left(n^{-1}\right), & X_{i} \leq \bar{X} \\ \sum_{\bar{X}_{(-i)}<X_{j}<\bar{X}} X_{j}=N\left(\triangle \mathbf{X}_{i}\right) \bar{X}+O_{p}\left(n^{-1}\right), & X_{i}>\bar{X}\end{cases}
$$


Combining the above results, we have

$$
\begin{aligned}
& \widehat{\hat{\theta}_{n, j a c k}} \\
= & \frac{1}{n} \sum_{i=1}^{n}\left\{n \hat{\theta}-(n-1) \hat{\theta}_{n-1}^{(-i)}\right\} \\
= & 2\left(N(\mathbf{X}) \bar{X}-\sum_{X_{j}<\bar{X}} X_{j}\right)-\frac{2}{n} \sum_{i=1}^{n}\left(N\left(\mathbf{X}_{i}\right) \bar{X}_{(-i)}-\sum_{X_{j}<\bar{X}_{(-i)}, i \neq j} X_{j}\right) \\
= & \frac{2 N(\mathbf{X}) \bar{X}}{n}-\frac{2}{n} \sum_{X_{i}<\bar{X}}^{n} X_{i}+\frac{2 \bar{X}}{n} \sum_{X_{i}>\bar{X}} N\left(\triangle \mathbf{X}_{i}\right)-\frac{2}{n} \sum_{X_{i}>\bar{X}}^{n}\left(\sum_{\bar{X}_{(-i)}<X_{j}<\bar{X}} X_{j}\right) \\
& -\frac{2 \bar{X}}{n} \sum_{X_{i}<\bar{X}} N\left(\triangle \mathbf{X}_{i}\right)+\frac{2}{n} \sum_{X_{i}<\bar{X}}^{n}\left(\sum_{\bar{X}<X_{j}<\bar{X}_{(-i)}} X_{j}\right)+o_{p}\left(n^{-1 / 2}\right) \\
= & \frac{2 N(\mathbf{X}) \bar{X}}{n}-\frac{2}{n} \sum_{X_{i}<\bar{X}}^{n} X_{i}+o_{p}\left(n^{-1 / 2}\right) .
\end{aligned}
$$

Thus, we have

$$
\sqrt{n}\left\{\widehat{\hat{\theta}_{n, j a c k}}-\theta\right\} \stackrel{\mathfrak{D}}{\longrightarrow} N\left(0, v^{2}\right) .
$$

Proof of Lemma 2.2:

$$
\begin{aligned}
\sigma_{n, j a c k}^{2} & =\frac{1}{n} \sum_{i=1}^{n}\left(\hat{V}_{i}-\theta\right)^{2} \\
& =\frac{1}{n} \sum_{i=1}^{n} \hat{V}_{i}^{2}-2 \theta \widehat{\hat{\theta}_{n, j a c k}}+\theta^{2} \\
& =\frac{1}{n} \sum_{i=1}^{n} \hat{V}_{i}^{2}-\theta^{2}+o_{p}(1) .
\end{aligned}
$$


From the above equations, we know that

$$
\begin{aligned}
& \frac{1}{4 n} \sum_{i=1}^{n} \hat{V}_{i}^{2} \\
& =\underbrace{\frac{1}{n} \sum_{i=1}^{n}\left\{N(\mathbf{X}) \bar{X}-N\left(\mathbf{X}_{i}\right) \bar{X}_{(-i)}\right\}^{2}}_{\mathrm{I}}+\underbrace{\frac{1}{n} \sum_{i=1}^{n}\left\{\sum_{X_{j}<\bar{X}} X_{j}-\sum_{X_{j}<\bar{X}_{(-i)}, i \neq j} X_{j}\right\}^{2}}_{\mathrm{II}} \\
& \underbrace{-\frac{2}{n} \sum_{i=1}^{n}\left\{N(\mathbf{X}) \bar{X}-N\left(\mathbf{X}_{i}\right) \bar{X}_{(-i)}\right\}\left\{\sum_{X_{j}<\bar{X}} X_{j}-\sum_{X_{j}<\bar{X}_{(-i)}, i \neq j} X_{j}\right\}}_{\text {III }} .
\end{aligned}
$$

Then, we investigate I, II and III, respectively.

$$
\begin{aligned}
\mathrm{I}= & \frac{1}{n} \sum_{i=1}^{n}\left\{N(\mathbf{X}) \bar{X}-N\left(\mathbf{X}_{i}\right) \bar{X}+N\left(\mathbf{X}_{i}\right) \frac{X_{i}-\bar{X}}{n-1}\right\}^{2} \\
= & \frac{1}{n} \sum_{i=1}^{n}\left\{\frac{N\left(\mathbf{X}_{i}\right)}{n-1}\left(X_{i}-\bar{X}\right)\right\}^{2}+\frac{1}{n} \sum_{X_{i}>\bar{X}}^{n} \bar{X}^{2}\left\{N\left(\triangle \mathbf{X}_{i}\right)\right\}^{2}+\underbrace{\frac{1}{n} \sum_{X_{i}<\bar{X}}^{n} \bar{X}^{2}\left\{1-N\left(\triangle \mathbf{X}_{i}\right)\right\}^{2}}_{\mathrm{IV}} \\
& +\underbrace{\frac{2}{n} \sum_{X_{i}<\bar{X}}^{n} \bar{X}\left\{1-N\left(\triangle \mathbf{X}_{i}\right)\right\} \frac{N\left(\mathbf{X}_{i}\right)}{n-1}\left(X_{i}-\bar{X}\right)}_{\mathrm{V}}+\frac{2}{n} \sum_{X_{i}>\bar{X}}^{n} \bar{X} N\left(\triangle \mathbf{X}_{i}\right) \frac{N\left(\mathbf{X}_{i}\right)}{n-1}\left(X_{i}-\bar{X}\right),
\end{aligned}
$$

and

$$
\mathrm{II}=\frac{1}{n} \sum_{X_{i}>\bar{X}}^{n}\left(\sum_{\bar{X}_{(-i)}<X_{j}<\bar{X}} X_{j}\right)^{2}+\underbrace{\frac{1}{n} \sum_{X_{i}<\bar{X}}^{n}\left(X_{i}-\sum_{\bar{X}<X_{j}<\bar{X}_{(-i)}} X_{j}\right)^{2}}_{\mathrm{VI}}
$$


and

$$
\begin{aligned}
& \mathrm{III}=\frac{2}{n} \sum_{X_{i}>\bar{X}}^{n}\left(\sum_{\bar{X}_{(-i)}<X_{j}<\bar{X}} X_{j}\right)\left\{\bar{X} N\left(\triangle \mathbf{X}_{i}\right)+N\left(\mathbf{X}_{i}\right) \frac{X_{i}-\bar{X}}{n-1}\right\} \\
& +\frac{2}{n} \sum_{X_{i}<\bar{X}}^{n}\left(X_{i}-\sum_{\bar{X}<X_{j}<\bar{X}_{(-i)}} X_{j}\right)\left\{\bar{X}\left\{1-N\left(\triangle \mathbf{X}_{i}\right)\right\}+N\left(\mathbf{X}_{i}\right) \frac{X_{i}-\bar{X}}{n-1}\right\} \\
& =\frac{2}{n} \sum_{X_{i}>\bar{X}}^{n}\left(\sum_{\bar{X}_{(-i)}<X_{j}<\bar{X}} X_{j}\right)\left\{\bar{X} N\left(\triangle \mathbf{X}_{i}\right)\right\}+\frac{2}{n} \sum_{X_{i}>\bar{X}}^{n}\left(\sum_{\bar{X}_{(-i)}<X_{j}<\bar{X}} X_{j}\right)\left\{N\left(\mathbf{X}_{i}\right) \frac{X_{i}-\bar{X}}{n-1}\right\} \\
& +\underbrace{\frac{2}{n} \sum_{X_{i}<\bar{X}}^{n}\left(X_{i}-\sum_{\bar{X}<X_{j}<\bar{X}_{(-i)}} X_{j}\right)\left\{\bar{X}\left\{1-N\left(\triangle \mathbf{X}_{i}\right)\right\}\right\}}_{\text {VII }} \\
& +\underbrace{\frac{2}{n} \sum_{X_{i}<\bar{X}}^{n}\left(X_{i}-\sum_{\bar{X}<X_{j}<\bar{X}_{(-i)}} X_{j}\right)\left\{N\left(\mathbf{X}_{i}\right) \frac{X_{i}-\bar{X}}{n-1}\right\}}_{\text {VIII }} .
\end{aligned}
$$

Considering IV, VI and VII, one has

$$
\begin{aligned}
& \mathrm{IV}+\mathrm{VI}-\mathrm{VII} \\
= & \frac{1}{n} \sum_{X_{i}<\bar{X}}^{n} \bar{X}\left\{1-N\left(\triangle \mathbf{X}_{i}\right)\right\}\left[\bar{X}\left\{1-N\left(\triangle \mathbf{X}_{i}\right)\right\}-\left(X_{i}-\sum_{\bar{X}<X_{j}<\bar{X}_{(-i)}} X_{j}\right)\right] \\
& -\frac{1}{n} \sum_{X_{i}<\bar{X}}^{n}\left(X_{i}-\sum_{\bar{X}<X_{j}<\bar{X}_{(-i)}} X_{j}\right)\left[\bar{X}\left\{1-N\left(\triangle \mathbf{X}_{i}\right)\right\}-\left(X_{i}-\sum_{\bar{X}<X_{j}<\bar{X}_{(-i)}} X_{j}\right)\right] \\
= & \frac{1}{n} \sum_{X_{i}<\bar{X}}^{n}\left[\bar{X}-X_{i}\right]^{2}+o_{p}(1) .
\end{aligned}
$$


From V and VIII, we have

$$
\begin{aligned}
& \mathrm{V}+\mathrm{VIII} \\
= & -\frac{2}{n} \sum_{X_{i}<\bar{X}}^{n}\left[\bar{X}-X_{i}\right]^{2} \frac{N\left(\mathbf{X}_{i}\right)}{n-1}+\frac{2}{n} \sum_{X_{i}<\bar{X}}^{n}\left\{\bar{X} N\left(\triangle \mathbf{X}_{i}\right)-\sum_{\bar{X}<X_{j}<\bar{X}_{(-i)}} X_{j}\right\} \frac{N\left(\mathbf{X}_{i}\right)}{n-1}\left(X_{i}-\bar{X}\right) \\
= & -\frac{2}{n} \sum_{X_{i}<\bar{X}}^{n}\left[\bar{X}-X_{i}\right]^{2} \frac{N\left(\mathbf{X}_{i}\right)}{n-1}+o_{p}(1) .
\end{aligned}
$$

Hence, we know

$$
\begin{aligned}
& \mathrm{I}+\mathrm{II}-\mathrm{III} \\
= & \frac{1}{n} \sum_{i=1}^{n}\left\{\frac{N\left(\mathbf{X}_{i}\right)}{n-1}\left(X_{i}-\bar{X}\right)\right\}^{2}-\frac{2}{n} \sum_{X_{i}<\bar{X}}^{n}\left[\bar{X}-X_{i}\right]^{2} \frac{N\left(\mathbf{X}_{i}\right)}{n-1}+\frac{1}{n} \sum_{X_{i}<\bar{X}}^{n}\left[\bar{X}-X_{i}\right]^{2}+o_{p}(1) \\
= & \frac{1}{n} \sum_{X_{i}<\bar{X}}^{n}\left\{\left[1-\frac{N\left(\mathbf{X}_{i}\right)}{n-1}\right]\left(X_{i}-\bar{X}\right)\right\}^{2}+\frac{1}{n} \sum_{X_{i}>\bar{X}}^{n}\left\{\frac{N\left(\mathbf{X}_{i}\right)}{n-1}\left(X_{i}-\bar{X}\right)\right\}^{2}+o_{p}(1) \\
= & \frac{v^{2}}{4}+o_{p}(1),
\end{aligned}
$$

which proves

$$
\sigma_{n, j a c k}^{2} \stackrel{\mathcal{P}}{\longrightarrow} v^{2}
$$

Proof of Theorem 1: Combining Lemmas 2.1 and 2.2, the standard arguments in Owen (1990), we prove Theorem 1.

Proof of Theorem 2: Like the procedure in Chen et al. (2008), we can obtain Theorem 2 from Theorem 1. Denote $Z_{n}=\max _{1 \leq i \leq n}\left|\widehat{V}_{i}-\theta_{0}\right|$. Using the result of 
Theorem 1 and equation (2.5), we have

$$
\begin{aligned}
0 & =\frac{1}{n} \sum_{i=1}^{n+1} g_{i}^{a d}\left(\theta_{0}\right)-\frac{\lambda}{n} \sum_{i=1}^{n+1} \frac{g_{i}^{a d}\left(\theta_{0}\right)^{2}}{1+\lambda g_{i}^{a d}\left(\theta_{0}\right)} \\
& \leq \bar{g}_{n}\left(\theta_{0}\right)\left(1-a_{n} / n\right)-\frac{\lambda}{n\left(1+\lambda \max _{1 \leq i \leq n}\left|\widehat{V}_{i}-\theta_{0}\right|\right)} \sum_{i=1}^{n}\left(\widehat{V}_{i}-\theta_{0}\right)^{2} \\
& =\bar{g}_{n}\left(\theta_{0}\right)-\frac{\lambda \sigma_{n, j a c k}^{2}}{1+\lambda Z_{n}}+O_{p}\left(n^{-3 / 2} a_{n}\right) .
\end{aligned}
$$

Thus, $\lambda=O_{p}\left(n^{-1 / 2}\right)$, as $a_{n}=o_{p}(n)$. Moreover, $\lambda=\left(\sigma_{n, j a c k}^{2}\right)^{-1} \bar{g}_{n}\left(\theta_{0}\right)+o_{p}\left(n^{-1 / 2}\right)$. The adjusted JEL ratio is as follows.

$$
\begin{aligned}
l^{a d}\left(\theta_{0}\right) & =2 \sum_{i=1}^{n+1}\left\{\lambda g_{i}^{a d}\left(\theta_{0}\right)-\lambda^{2}\left(g_{i}^{a d}\right)^{2}\left(\theta_{0}\right) / 2\right\}+o_{p}(1) \\
& =n\left(\sigma_{n, j a c k}^{2}\right)^{-1} \bar{g}_{n}^{2}\left(\theta_{0}\right)+o_{p}(1) \\
& \stackrel{d}{\rightarrow} \chi_{1}^{2}
\end{aligned}
$$

Proof of Theorem 3: Based on the proof of theorems by Tsao and Wu (2013), we can give a proof of Theorem 3. As Tsao and Wu (2013), one can define

$$
h_{n}^{c}(\theta)=\hat{\theta}+\gamma(n, l(\theta))(\theta-\hat{\theta})
$$

where the expansion factor $\gamma(n, l(\theta))$ is defined before. Define the generalized inverse $h_{n}^{-c}(\theta)$ like Tsao and $\mathrm{Wu}(2013)$. Thus, the extended EL ratio $l^{*}(\theta)=l\left(h_{n}^{-c}\right)$. For any any $\theta$ lies in $\left\{\theta:\left\|\theta-\theta_{0}\right\| \leq n^{-1 / 2}\right\}$, we apply Taylor expansion,

$$
l(\theta)=l\left(\theta_{0}+\theta-\theta_{0}\right)=l\left(\theta_{0}\right)+\frac{\partial l\left(\theta_{0}\right)}{\partial \theta}\left(\theta-\theta_{0}\right)+O_{p}\left(n^{-1}\right),
$$

where $\partial l\left(\theta_{0}\right) / \partial \theta=O_{p}\left(n^{1 / 2}\right)$ and $l(\theta)=O_{p}(1)$. For $\widetilde{\theta}_{0}=h_{n}^{-c}(\theta)$, it is clear that $\widetilde{\theta}_{0}-\theta_{0}=$ $O_{p}\left(n^{-3 / 2}\right)$ and $\widetilde{\theta}_{0} \in\left[\hat{\theta}, \theta_{0}\right]$ as the arguments in Tsao and $\mathrm{Wu}(2013)$. Hence we have 
$l^{*}\left(\theta_{0}\right)=l\left(h_{n}^{-c}\right)=l\left(\widetilde{\theta}_{0}\right)$. Moreover, we obtain by Taylor expansion

$$
l^{a d}\left(\theta_{0}\right)=l\left(\theta_{0}\right)+\frac{\partial l\left(\theta_{0}\right)}{\partial \theta}\left(\widetilde{\theta}-\theta_{0}\right)+O_{p}\left(n^{-1}\right)=l\left(\theta_{0}\right)+O_{p}\left(n^{-1}\right)
$$

Then, the extended JEL ratio $l^{a d}\left(\theta_{0}\right)$ has the same asymptotic distribution as the JEL ratio $l\left(\theta_{0}\right)$. 
Table 1: Coverage probability (average length) of confidence intervals under a normal distribution

\begin{tabular}{clcccc}
\hline \hline$n$ & $\begin{array}{l}\text { Nominal } \\
\text { Level }\end{array}$ & NA & JEL & AJEL & EJEL \\
\hline \multirow{3}{*}{30} & .99 & $.852(.507)$ & $.982(.583)$ & $.986(.585)$ & $.996(.584)$ \\
& .95 & $.816(.386)$ & $.930(.446)$ & $.946(.442)$ & $.951(.448)$ \\
& .90 & $.790(.324)$ & $.874(.367)$ & $.897(.366)$ & $.895(.371)$ \\
\hline \multirow{3}{*}{50} & .99 & $.904(.411)$ & $.987(.456)$ & $.990(.446)$ & $.999(.447)$ \\
& .95 & $.864(.313)$ & $.943(.340)$ & $.956(.340)$ & $.951(.339)$ \\
& .90 & $.831(.263)$ & $.888(.281)$ & $.904(.283)$ & $.896(.283)$ \\
\hline \multirow{3}{*}{100} & .99 & $.947(.303)$ & $.987(.316)$ & $.991(.313)$ & $.988(.316)$ \\
& .95 & $.905(.230)$ & $.948(.239)$ & $.951(.237)$ & $.949(.238)$ \\
& .90 & $.861(.193)$ & $.898(.200)$ & $.905(.198)$ & $.902(.199)$ \\
\hline \multirow{3}{*}{200} & .99 & $.969(.217)$ & $.990(.222)$ & $.991(.222)$ & $.991(.221)$ \\
& .95 & $.930(.165)$ & $.953(.169)$ & $.958(.168)$ & $.953(.168)$ \\
& .90 & $.887(.138)$ & $.901(.141)$ & $.906(.140)$ & $.902(.141)$ \\
\hline \multirow{3}{*}{300} & .99 & $.976(.178)$ & $.990(.181)$ & $.990(.180)$ & $.990(.181)$ \\
& .95 & $.938(.135)$ & $.951(.137)$ & $.954(.137)$ & $.952(.137)$ \\
& .90 & $.889(.114)$ & $.906(.115)$ & $.912(.115)$ & $.907(.115)$ \\
\hline \hline
\end{tabular}


Table 2: Coverage probability (average length) of confidence intervals under an exponential distribution

\begin{tabular}{cccccc}
\hline \hline$n$ & $\begin{array}{l}\text { Nominal } \\
\text { Level }\end{array}$ & NA & JEL & AJEL & EJEL \\
\hline \multirow{3}{*}{30} & .99 & $.735(.447)$ & $.982(.783)$ & $.995(.787)$ & $.989(.770)$ \\
& .95 & $.697(.333)$ & $.920(.619)$ & $.943(.565)$ & $.950(.595)$ \\
& .90 & $.668(.362)$ & $.860(.502)$ & $.892(.491)$ & $.878(.490)$ \\
\hline \multirow{3}{*}{50} & .99 & $.806(.343)$ & $.975(.635)$ & $.982(.617)$ & $.987(.624)$ \\
& .95 & $.765(.260)$ & $.924(.488)$ & $.938(.485)$ & $.936(.474)$ \\
& .90 & $.722(.221)$ & $.871(.395)$ & $.890(.398)$ & $.871(.392)$ \\
\hline \multirow{3}{*}{100} & .99 & $.856(.342)$ & $.980(.470)$ & $.984(.466)$ & $.984(.469)$ \\
& .95 & $.805(.273)$ & $.930(.350)$ & $.941(.349)$ & $.931(.345)$ \\
& .90 & $.751(.220)$ & $.876(.291)$ & $.886(.289)$ & $.875(.290)$ \\
\hline \multirow{3}{*}{200} & .99 & $.909(.343)$ & $.983(.331)$ & $.986(.330)$ & $.984(.332)$ \\
& .95 & $.846(.262)$ & $.942(.247)$ & $.948(.247)$ & $.942(.246)$ \\
& .90 & $.783(.221)$ & $.890(.206)$ & $.898(.206)$ & $.890(.205)$ \\
\hline \multirow{3}{*}{300} & .99 & $.918(.342)$ & $.985(.269)$ & $.987(.269)$ & $.984(.268)$ \\
& .95 & $.849(.262)$ & $.936(.202)$ & $.940(.202)$ & $.937(.201)$ \\
& .90 & $.782(.225)$ & $.879(.169)$ & $.887(.168)$ & $.881(.168)$ \\
\hline \hline
\end{tabular}


Table 3: Length of confidence intervals with the hot dogs data set

\begin{tabular}{|c|c|c|c|c|c|c|c|c|c|}
\hline Nominal & & $N A$ & & $\overline{J E L}$ & & $\overline{A J E L}$ & & $\overline{E J E L}$ & \\
\hline Level & & UB & LB & UB & LB & UB & LB & UB & LB \\
\hline \multirow{3}{*}{.99} & & 96.815 & 58.469 & 99.392 & 60.101 & 100.476 & 61.365 & 99.283 & 60.189 \\
\hline & Length & 38.346 & & 39.291 & & 39.111 & & 39.094 & \\
\hline & & 92.230 & 63.053 & 93.411 & 63.941 & 94.605 & 65.238 & 93.331 & 64.011 \\
\hline \multirow[t]{2}{*}{.95} & Length & 29.177 & & 29.470 & & 29.367 & & 29.320 & \\
\hline & & 89.885 & 65.399 & 90.535 & 65.956 & 91.773 & 67.268 & 90.470 & 66.015 \\
\hline .90 & Length & 24.486 & & 24.579 & & 24.505 & & 24.455 & \\
\hline
\end{tabular}


Table 4: Length of confidence intervals with the discoveries data set

\begin{tabular}{|c|c|c|c|c|c|c|c|c|c|}
\hline Nominal & & $N A$ & & $J E L$ & & $A J E L$ & & $E J E L$ & \\
\hline Level & & UB & LB & UB & LB & UB & LB & UB & LB \\
\hline \multirow{3}{*}{.99} & & 2.007 & 1.341 & 2.352 & 1.268 & 2.343 & 1.270 & 2.355 & 1.267 \\
\hline & Length & 0.666 & & 1.084 & & 1.073 & & 1.088 & \\
\hline & & 1.928 & 1.421 & 2.163 & 1.356 & 2.156 & 1.357 & 2.164 & 1.355 \\
\hline \multirow[t]{2}{*}{.95} & Length & 0.507 & & 0.807 & & 0.799 & & 0.809 & \\
\hline & & 1.887 & 1.461 & 2.074 & 1.403 & 2.068 & 1.404 & 2.075 & 1.402 \\
\hline .90 & Length & 0.426 & & 0.671 & & 0.664 & & 0.673 & \\
\hline
\end{tabular}

